\title{
Facilitating Early Integration of Palliative Care into Breast Cancer Therapy. Promoting Disease-Specific Guidelines
}

\author{
Jan Gaertner ${ }^{\mathrm{a}, \mathrm{b}}$ Rachel Wuerstlein ${ }^{\mathrm{b}, \mathrm{c}}$ Christoph Ostgathe $\mathrm{e}^{\mathrm{a}, \mathrm{b}}$ \\ Peter Mallmann ${ }^{\mathrm{b}, \mathrm{c}}$ Nadia Harbeck ${ }^{\mathrm{b}, \mathrm{c}}$ Raymond Voltz ${ }^{\mathrm{a}, \mathrm{b}}$ \\ aDepartment of Palliative Medicine, University Hospital Cologne, \\ ${ }^{b}$ Center for Integrated Oncology Köln/Bonn, \\ 'Department of Senology, University Hospital Cologne, Germany
}

\section{Keywords}

Comprehensive cancer care - Palliative medicine .

Early integration - Quality of Life

\section{Summary}

To comply with patients' needs as well as ASCO and WHO recommendations, our institution aims to integrate palliative care $(P C)$ early in the course of breast cancer (BC) therapy. The evaluation of relevant pilot project data revealed that these recommendations were too vague to trigger $\mathrm{PC}$ integration. Therefore, a standard operating procedure (SOP) was developed by our interdisciplinary working group to provide disease-specific information to overcome the ambiguity of the WHO recommendations and guide $\mathrm{PC}$ integration. Literally, the SOP states that 'Specialized PC is recommended regularly for all BC patients without curative treatment options, specifically for patients with i) metastasized and inoperable, or ii) locally advanced and inoperable, or iii) relapsing $\mathrm{BC}$, who are receiving intravenous chemotherapy'. This SOP for the first time presents disease-specific guidelines for $P C$ integration into comprehensive $B C$ therapy by defining 'green flags' for early integration of $\mathrm{PC}$ and delineating PC from senology assignments. Although disease-specific SOPs have also been developed by this working group for other malignancies, the decision when to first integrate PC into BC therapy differs substantially because of the different clinical characteristics of the disease.

\author{
Schlüsselwörter \\ Comprehensive Cancer Care · Palliativmedizin . \\ Frühe Integration · Lebensqualität
}

\section{Zusammenfassung}

Um den Bedürfnissen der Patientinnen ebenso zu entsprechen wie den aktuellen ASCO- und WHO-Empfehlungen, versucht unsere Institution, Palliative Care (PC) früh in den Verlauf der Brustkrebstherapie zu integrieren. Die Auswertung relevanter Pilotprojektdaten zeigte, dass die Empfehlungen der WHO zu vage waren, um PC-Integration auszulösen. Daher wurde von unserer interdisziplinären Arbeitsgruppe eine Standard Operating Procedure (SOP) entwickelt, um durch spezifischere Informationen die Uneindeutigkeit der WHO-Empfehlungen zu überwinden. Konkret empfiehlt diese SOP die regelmäßige Mitbetreuung durch spezialisierte PC für alle Brustkrebs-Patientinnen ohne kurative Behandlungsoptionen. Dies gilt insbesondere für Patientinnen mit i) metastasiertem und inoperablen Brustkrebs oder ii) lokal fortgeschrittenem und inoperablen Brustkrebs oder iii) einem Rezidiv der Erkrankung, sobald diese Patienten intravenöse, systemische Chemotherapie erhalten. Diese SOP präsentiert erstmalig krankheitsspezifische Leitlinien für die Integration von PC in die Brustkrebstherapie durch die Definition von "green flags" und die Klärung der Zuständigkeiten von PC und Senologie. Aufgrund des unterschiedlichen Krankheitsverlaufs unterscheidet sich die Brustkrebs-SOP wesentlich von SOPs für andere solide Tumoren. Dies gilt insbesondere für die Definition des Zeitpunkts der PC-Integration.

\section{KARGER \\ Fax +497614520714 \\ Information@Karger.de}

www.karger.com (c) 2011 S. Karger GmbH, Freiburg

$1661-3791 / 11 / 0063-0240 \$ 38.00 / 0$

Accessible online at:

www.karger.com/brc 


\section{Introduction}

Palliative care (PC) is one of the fastest growing medical specialties. Current World Health Organization (WHO) recommendations demand the integration of PC early in the course of life-threatening illness, especially cancer [1] (fig. 1). This contradicts older, meanwhile outdated, clinical pathways when 'transition' to PC took place in the very last stages of the disease $[2,3]$. The concept recommended by the WHO and most international health services demands that patients with incurable cancer should have access to specialized PC competence and infrastructure earlier in the course of disease in addition to oncologic therapy [4-7]. For the treatment of cancer patients, recently published American Society of Clinical Oncology (ASCO) guidelines affirm this recommendation by demanding the implementation of specialized PC programs into cancer therapy from the time of diagnosis or presentation of an incurable malignancy [8] (fig. 2).

Our institution is dedicated to delivering state-of-the-art comprehensive cancer care. Therefore, all academically independent departments that are involved in the care for cancer patients cooperate as part of our Comprehensive Cancer Center (CIO). Therefore, the Department of Senology and all other relevant departments such as Radiotherapy, Surgery, Psychooncology, and Palliative Medicine are involved in patient care according to international standards and patient needs. Therefore, to follow the WHO recommendation for early integration of PC, the CIO decided i) to include this recommendation into the administrative directives for principles of cancer care, and ii) to establish a PC hospital support team (PCST) following the European Commission recommendations to enable early integration into cancer disease trajectories [9]. The PCST was set up in addition to the existing inpatient and home-specialized PC service.

The evaluation of relevant pilot project data revealed that this approach was too vague to serve as a general clinical guideline to successfully implement early integration of PC $[10,11]$. As a consequence, in addition to inclusion in administrative directives and establishment of a PCST, we decided to develop standard operating procedures (SOPs) as diseasespecific guidelines to i) define disease-specific points in the

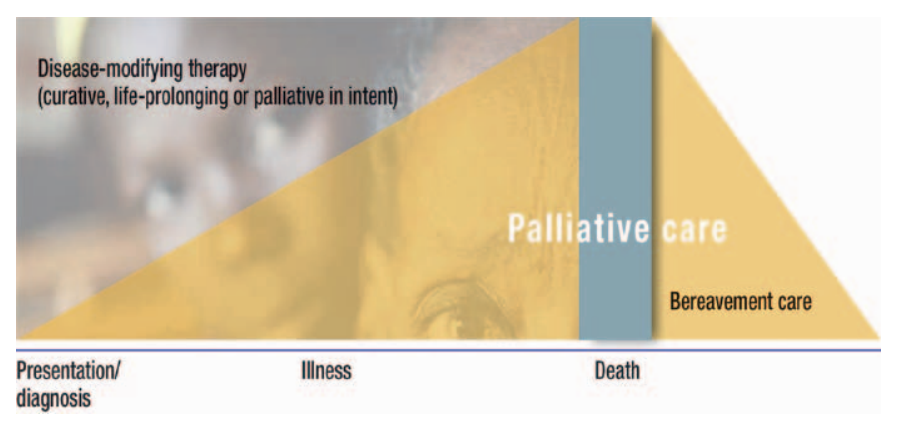

Fig. 1. Integrated model of palliative care into the course of chronic progressive illness (source: WHO, with permission [1]). disease trajectory when PC should be integrated into the clinical pathway, and ii) provide concise definitions of PC structure and competencies. Paradigmatically, the SOP for comprehensive breast cancer therapy is presented here, since its therapeutic algorithm differs substantially from the SOPs for other solid tumors such as lung cancer $[10,12]$ because of the clinical characteristics of the disease.

\section{Text of the Consensus SOP Breast Cancer}

\section{Background Information}

Patients with i) metastasized and inoperable or ii) locally advanced and inoperable breast cancer may have a survival time of several years. The course of the disease is hard to predict and can sometimes span several decades. As a result of novel treatment options according to innovative clinical study protocols, survival time is expected to increase further. Still, patients often suffer from burdening symptoms and psychosocial burden requiring specialized PC. Analogous to the WHO and ASCO recommendations for $\mathrm{PC}$, the integration of $\mathrm{PC}$ is advocated early in the course of BC therapy. Reasons for this are i) attainment of best possible symptom control and quality of life through conjoint interdisciplinary treatment, ii) provision of best possible cross-sectoral PC infrastructure to optimize home care and iii) comprehensive care for patients and proxies also in psychosocial and spiritual issues, iv) prevention of patients and proxies feeling abandoned after tumorspecific treatment, v) provision of information for patients and families as to where to obtain specific support in the case of problematic situations or symptoms in addition to the primarily treating department, and vi) support of the primarily treating oncologic department.

\section{Time and Place of PC Integration}

Specialized PC is recommended regularly for all breast cancer patients without curative treatment options, specifically patients with i) metastasized and inoperable breast cancer, or ii) locally advanced and inoperable breast cancer, or iii) relapsing breast cancer, who are receiving intravenous chemotherapy. Integration of $\mathrm{PC}$ is recommended soon after the

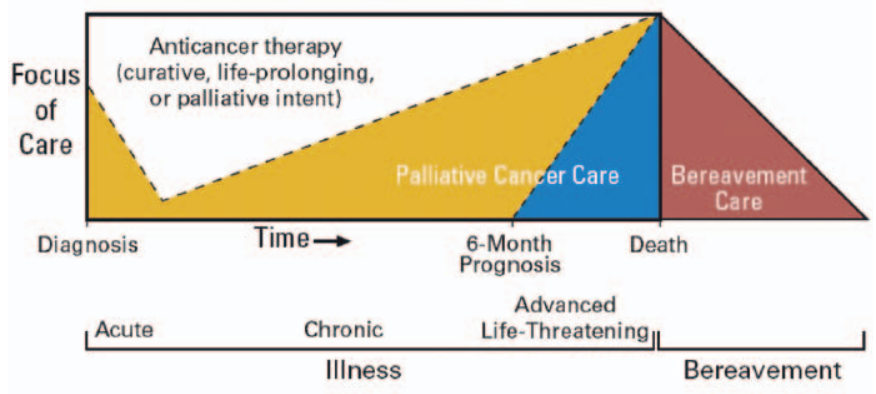

Fig. 2. ASCO approach of integration of specialized palliative care (with permission [8]). 
start of tumor-specific therapy. In this context, initial PC should be delivered to the patient in the same place as specific treatment, which is the interdisciplinary outpatient unit of the Department of Senology.

\section{Terminology and Infrastructure}

PC assignments do not include supportive measures (treatment of side effects of tumor-specific therapy) or 'palliative therapies'. These are tumor-specific interventions (e.g. systemic, radiotherapeutic, or surgical intervention) which primarily aim at prolonging life by modulating the progress of the disease. These therapeutic interventions are delivered by the particular specialty (e.g. hematooncology, radiotherapy, surgery). Depending on the patient's needs, the Department of Palliative Medicine provides an inpatient unit, a day clinic, a consulting service, and a palliative home care team.

\section{Discussion}

Is the WHO Recommendation Helpful in the Implementation of Early Integration of PC?

The necessity of this early integration of PC in the course of any life-limiting disease has become widely accepted among PC providers [13-15]. The WHO states that ' $\mathrm{PC}$ is applicable early in the course of illness, in conjunction with other therapies that are intended to prolong life, such as chemotherapy or radiation therapy' [1]. However, the recommendation does not contain any further suggestions about how this could be accomplished. Despite this shortcoming, our institution decided to i) include this recommendation in the administrative directives for principles of cancer care, and ii) establish a PCST following the European Commission recommendations to enable early integration into cancer disease trajectories [9]. The PCST was set up in addition to the existing inpatient and home-specialized PC service. To evaluate the experience of the first 2 years of routine early integration, the relevant data were analyzed [11]. The main finding of this evaluation was that our approach did not suffice to implement early integration. Specifically, despite the recommendation described above and the provision of a cross-sectoral PC infrastructure including a PCST, most patients were seen rather late in the course of the disease. This was reflected by the fact that at the time of the first contact with the PCST, they already had a reduced performance status or suffered from symptoms often associated with advance disease (e.g. dyspnea) [11]. This is in line with the statement of the National Comprehensive Cancer Network (NCCN) who reported about the difficulty of implementing PC guidelines into everyday practice $[7,16]$, as well as our own findings from assessing PC integration into lung cancer therapy [10].

\section{Decision for Disease-Specific PC SOPs}

Because of the experiences described above, we decided that the sole adoption of the WHO recommendation in the administrative guidelines plus the provision of a PCST in addition to the preexisting home care service and inpatient ward did not suffice to integrate PC early enough. Therefore, we decided to develop a PC SOP that would be adopted disease-specifically for integration into our 24 cancer therapy SOPs that had been developed previously for the vast majority of hematooncologic malignancies. This approach differs from the NCCN concept which tries to trigger early PC integration by the expected survival time or symptom burden of the patients [7, 16]. Yet, correctly identifying the expected survival time of a specific patient is often problematic $[17,18]$, and using only symptom burden as a trigger for PC integration may lead to (too) late referrals $[19,20]$. Therefore, we decided to go for the development of a disease-specific SOP. Such guidelines should provide disease- and stage-specific points to institutionalize and ensure early integration of PC and provide information about PC assignments and competencies within the comprehensive cancer setting.

\section{When Should Specialized PC Be Integrated (Green Flags)?}

The SOP for breast cancer differs substantially from SOPs developed for other malignancies in our center such as lung cancer $[10,12]$. Because of the unique course of the disease that may last for decades, we considered it inappropriate to integrate PC right after diagnosing a metastasized illness. On the other hand, the integration should not take place too late in the disease trajectory. For example, if a patient's first contact to a PC physician is arranged when no other options of systemic or local therapy aiming at cancer 'control' remain, this is often perceived as a sudden 'switch' of the therapeutic goals and patients experience fear and a feeling of being abandoned [10, 21]. Therefore, following interdisciplinary discussions, another approach was chosen. Since after initiation of intravenous chemotherapy the prognosis of patients with metastasized disease is limited substantially, to our interdisciplinary working group, this appeared to be the most adequate point in the disease trajectory to routinely integrate specialized PC into patient care. The SOP for breast cancer literally recommends the initiation of $\mathrm{PC}$ 'straight after completion of staging at defined stages of the disease; specifically, PC integration is advised for patients with i) locally advanced and inoperable breast cancer, ii) metastasized breast cancer, or iii) relapsed breast cancer, who are receiving intravenous chemotherapy'. By this, it provides a clear algorithm for when to integrate PC. This is necessary since despite factual knowledge many clinicians are still afraid that cancer patients and their relatives might be discouraged after talking to PC professionals [22, 23]. Others do not perceive that patients and their families benefit from the early integration of PC structures in their treatment [24]. 
What PC Services Should Be Integrated?

Although it is acknowledged that the integration of PC is imperative for the treatment of cancer patients [10, 20], PC infrastructure has evolved differently around the globe [25]. While some countries traditionally support the development of palliative home care services, others focus on inpatient care such as hospice and/or PC units [5]. Others argue that the implementation of PC consulting teams (hospital support teams, PCST) best meets the needs of cancer patients [26]. To assure that appropriate PC is available at all stages of the disease, a cross-sectoral approach is being increasingly suggested [5]. Therefore, the SOP states that a PCST should be provided. The multiprofessional team consists of a qualified and specially trained PC nurse with longtime experience and a consultant PC specialist. The PCST is to meet the patient in the same location as the oncology team (e.g. within the comprehensive cancer care outpatient unit) to ensure a familiar environment and 'seamless' care. To coordinate the PC services, PC case management was established, and the PC staff routinely attends the interdisciplinary tumor board meetings. Outpatients can also be cared for by our PC-trained nurses and specialized PC physicians of the outpatient PC team to support general practitioners and home care services. The inpatient PC unit has proved to be an essential component of specialized PC infrastructures in providing adequate support for severely affected patients [22-24].

\section{Terminology}

As von Gunten [25] and Cherny [27] pointed out, a close look at terminology is crucial to successfully implement PC programs. As in other languages, in the German language it is necessary to clearly distinguish PC expertise from 'supportive therapy' or 'palliative therapy' to prevent watering down of $\mathrm{PC}$ assignments, or therapeutic confusion [2, 13, 25, 28]. It is becoming increasingly accepted that as treatment options for systemic cancer are increasing rapidly, it is crucial to clearly distinguish specialized PC assignments from mere oncology $[8,29]$. Therefore, the SOP explicitly states that 'PC assignments do not include supportive measures (treatment of side effects of tumor-specific therapy) or 'palliative therapies'. The latter are tumor-specific interventions (e.g. systemic or radiotherapy or surgical intervention) which primarily aim at prolonging life by modulating the progress of the disease. These therapeutic interventions are delivered by the particular specialty (e.g. hematooncology, radiotherapy, surgery)' (table 1).

\section{Perspective and Limitations}

Meanwhile, the interdisciplinary oncology working groups of 23 other hematooncologic diseases decided to develop disease-specific PC SOPs based on the SOP presented here.
Table 1. 'Flags'

\begin{tabular}{|c|c|}
\hline Green flags & $\begin{array}{l}\text { locally advanced and inoperable breast cancer } \\
\text { after initiation of systemic chemotherapy } \\
\text { metastasized and inoperable breast cancer } \\
\text { after initiation of systemic chemotherapy } \\
\text { relapsed breast cancer after initiation of systemic } \\
\text { chemotherapy } \\
\text { completion of staging, shortly after starting } \\
\text { tumor-specific therapy }\end{array}$ \\
\hline Red flags & $\begin{array}{l}\text { supportive measures: treatment of side effects } \\
\text { of tumor-specific therapy } \\
\text { 'palliative therapies': tumor-specific interventions } \\
\text { primarily aiming at prolonging life by modulating } \\
\text { the disease progress }\end{array}$ \\
\hline
\end{tabular}

To date, we are unable to provide data about the acceptability of this SOP and its impact on referrals to PC. Additionally detailed disease-independent guidelines for PC skills, attitudes, and knowledge are being developed. The authors hope to provide valuable information and initiate a discussion about the integration of specialized PC into the complex course of breast cancer therapy. Yet, beyond the provision of such SOPs, future developments are necessary. These should aim at i) implementing PC as a mandatory component of cancer therapy, ii) ensuring that beyond the mere provision of guidelines, PC attitude and culture becomes an integral part of patient care, and iii) maintaining a holistic approach to PC which should not be limited to treatment algorithms.

\section{Conclusions}

Disease trajectories and patient needs differ between different malignancies. While the identification of the point in the disease trajectory at which the disease is clearly 'life-threatening, incurable, and progressive' might be more intuitive for other solid tumors such lung cancer $[10,12]$, the identification of such therapeutic landmarks is more challenging for breast cancer, mainly because of its allotropic origin and extremely variable etiopathology. This SOP for the first time presents disease-specific guidelines for PC integration into comprehensive breast cancer therapy by i) defining 'green and red flags' for early integration of $\mathrm{PC}$, ii) recommending $\mathrm{PC}$ to be initiated parallel to anti-cancer therapy, and iii) specifying PC assignments and infrastructure.

\section{Disclosure Statement}

The authors declare no conflict of interest. 


\section{References}

1 WHO: National Cancer Control Programmes Policies and Managerial Guidelines, 2nd ed. Geneva, WHO,2002.

2 Hanks G: Palliative care: careless use of language undermines our identity. Palliat Med 2008;22:10910 .

$\checkmark 3$ Bruera E: The development of a palliative care culture. J Palliat Care 2004;20:316-9.

4 Higginson IJ, Costantini M: Dying with cancer, living well with advanced cancer. Eur J Cancer 2008;44:1414-24.

5 Higginson IJ: End-of-life care: lessons from other nations. J Palliat Med 2005;8(suppl 1):S161-73.

6 Higginson IJ, Finlay IG: Improving palliative care for cancer. Lancet Oncol 2003;4:73-4.

7 Levy MH, Back A, et al.: NCCN clinical practice guidelines in oncology: palliative care. J Natl Compr Canc Netw 2009;7:436-73.

8 Ferris FD, Bruera E, et al.: Palliative cancer care a decade later: accomplishments, the need, next steps - from the American Society of Clinical Oncology. J Clin Oncol 2009;27:3052-8.

9 European Commission: Promoting the development and integration of palliative care mobile support teams in the hospital - the fifth framework programme 1998-2002 'Quality of Life and Mangement of Living Resources. Brussels, European Commission, 2003.

10 Gaertner J, Wolf J, et al.: Implementing the WHO recommendation for palliative care into routine lung cancer therapy - a feasibility project. J Palliat Med 2010;13:727-32
11 Gaertner $\mathbf{J}$, Wolf $\mathbf{J}$, et al.: Integrating palliative medicine into comprehensive breast cancer therapy - a feasibility project. Breast Care 2011;Epub ahead of print.

12 Gaertner J, Wolf J, et al.: Specifying WHO recommendation - moving towards disease specific guidelines. J Palliat Med 2010;13:1273-6.

13 Periyakoil VS, von Gunten CF: Mainstreaming palliative care. J Palliat Med 2007;10:40-2.

14 Periyakoil VS, Hallenbeck J: Identifying and managing preparatory grief and depression at the end of life. Am Fam Physician 2002;65:883-90.

15 Von Gunten CF: Palliative medicine. Impact. J Palliat Med 2008;11:536-7.

$16 \mathrm{McNeil} \mathrm{C:} \mathrm{Can} \mathrm{guidelines} \mathrm{be} \mathrm{integrated} \mathrm{into} \mathrm{every-}$ day practice? The NCCN in year 4. National Comprehensive Cancer Network (news). J Natl Cancer Inst 1999;91:753-5.

17 Stiel S, Bertram L, et al.: Evaluation and comparison of two prognostic scores and the physicians' estimate of survival in terminally ill patients. Support Care Cancer 2009; Epub ahead of print

18 Liao S, Arnold RM: Prognosticating: the end of a series. Introduction. J Palliat Med 2008;11:82-3.

19 Temel JS, Jackson VA, et al.: Phase II study: integrated palliative care in newly diagnosed advanced non-small-cell lung cancer patients. J Clin Oncol 2007;25:2377-82.

20 Temel JS, Pirl WF, Lynch TJ: Comprehensive symptom management in patients with advancedstage non-small-cell lung cancer. Clin Lung Cancer 2006:7:241-9.
21 Quill TE: Dying and decision making - evolution of end-of-life options. N Engl J Med 2004; 350:2029-32.

22 Snow CE, Varela BR, et al.: Identifying factors affecting utilization of an inpatient palliative care service: a physician survey. J Palliat Med 2009;12:231-7.

23 Fadul N, Elsayem A, et al.: Predictors of access to palliative care services among patients who died at a Comprehensive Cancer Center. J Palliat Med 2007;10:1146-52.

24 Billings JA: Dignity. J Palliat Med 2008;11:138-9.

25 Von Gunten CF: Humpty-Dumpty syndrome. Palliat Med 2007;21:461-2.

26 Elsayem A, Smith ML, et al.: Impact of a palliative care service on in-hospital mortality in a comprehensive cancer center. J Palliat Med 2006;9:894 902.

27 Cherny NI: Stigma associated with 'palliative care': getting around it or getting over it. Cancer 2009;115:1808-12.

28 Von Gunten CF: The hidden (real) curriculum. J Palliat Med 2007;10:632-3.

29 Rayson D, McIntyre P: Transitions to palliation: two solitudes or inevitable integration? Curr Oncol Rep 2007;9:285-9. 